\title{
Instalando la ciudad del consumo: el palimpsesto urbano del primer shopping mall chileno en el fundo San Luis, Santiago
}

Rosa-Liliana De Simone. Pontificia Universidad Católica de Chile, Santiago, Chile.

RESUMEN | Como un palimpsesto histórico, las políticas urbanas y económicas dejan huellas en la ciudad y construyen discursos sobre imaginarios urbanos. Construido en el que fue el fundo San Luis entre 1979 y 1982, Parque Arauco Shopping Center fue presentado por los medios y sus promotores como una promesa cumplida del progreso económico traído por un nuevo gobierno, y como un modelo de vida suburbano inspirado en ideales de consumo de masas y automobilización. La relevancia de Parque Arauco en la historia urbana tiene que ver con la articulación discursiva de sus imaginarios sobre modernidad, su polémica instalación en tiempos de polarizaciones ideológicas y con su localización en un predio cargado de proyectos anteriores. El proceso de instalación del primer mall chileno puede ser estudiado como un acto simbólico que proclamó y materializó el giro ideológico de una ciudad hoy marcada por el consumo como una fuerza productora del espacio urbano.

PALABRAS CLAVE | consumo, planificación urbana, historia urbana.

ABSTRACT | Analogous to a historical palimpsest, urban and economic policies leave footprints in cities and build discourses about urban imaginaries. Built on the ex San Luis Estate between 1979 and 1982, Parque Arauco Shopping Center was promoted as an accomplished promise of the economic progress brought by a new government, and as a model for a suburban lifestyle inspired by mass consumption and motorization. Parque Arauco's significance in urban history holds relation with the discursive articulation of its imaginaries about modernity, its polemic installation in ideologically polarized times, and its localization in a site that was loaded with previous projects. The installment process of Parque Arauco can be studied as a symbolic act, which proclaimed and materialized the ideological turn in a city that is now defined by consumption as a productive force of urban space.

KEYWORDS | consumption, urban planning, urban history. 


\section{Introducción}

Parque Arauco Shopping Center se construyó en Santiago de Chile en tiempos de fuerte crisis económica, lo que matizó su arribo y suavizó sus críticas iniciales. Pese al contexto recesivo inicial, el mall fue edificando una imagen mediática que lo volvía rostro de la renovación económica en los ańos ochenta y de bonanza milagrosa en los noventa. Esto lo llevó a ser usado como un icono de los tiempos, tanto en su calidad de evidencia de éxito para la defensa del nuevo modelo económico como en cuanto constatación de la mercantilización de la vida social, ello por parte de la oposición política.

A través de discursos mediáticos, los gestores mostraron el mall como un producto de las reformas económicas impulsadas en 1975, un proyecto capaz de detonar cambios urbanos y económicos como casi ninguna otra infraestructura edilicia en la ciudad.

Contrarios al oficialismo, los intelectuales más ligados a la izquierda y a la oposición lo rechazaron como emblema de los "cambios estructurales" que se implementaban violentamente en el país. El consumo conspicuo no calzaba con los ideales de ascetismo cristiano que atravesaban buena parte de los discursos de la aristocracia chilena (Salcedo \& De Simone, 2013). Más allá, el arribo del mall también fue visto como la implantación forzada del modo de vida norteamericano (Iribarne $\&$ Friedmann, 1984), que desde los años cincuenta estaba siendo expandido en la zona latinoamericana a través de instituciones embajadoras y políticas diplomáticas que los Estados Unidos lideraban.

A pesar de las críticas, el retorno a la democracia en los noventa trajo también una búsqueda por democratizar aquellas estructuras que permanecieron exclusivas para unos pocos. Así, el mall se multiplicó en Santiago en los noventa y expandió por todo el país en los 2000, hasta posicionar a Chile como el líder en retail en Sudamérica y como el país con más metros cuadrados de retail por habitante en la región (A.T. Kearney, 2016). Este éxito no se explica solo por un buen modelo de negocios. Desde sus primeros ańos, los malls se llenaron de emocionados santiaguinos hasta convertirse en uno de los principales escenarios de vida urbana, siendo así integrados con éxito al imaginario citadino.

No obstante, el nuevo rol urbano de los malls importados en los noventa no fue una novedad; más bien, fue un perfil heredado de una férrea línea de centros comerciales de diseño chileno, que desde mediados de siglo inventaron formas de atraer a la población. Hablamos de los protomalls: patios, placas, pérgolas, caracoles y otros modelos de comercio vecinal de invención chilena que, si bien no cumplen con las exigencias que definen a un shopping mall como tal, anticiparon los usos y los ambientes que pavimentarían el camino de estos hacia el escenario urbano (De Simone, 2015).

¿Cómo se construyeron los discursos e imaginarios que instalaron la sociedad de consumo en la ciudad chilena? 
Figura I | Localización de Parque Arauco en la ciudad de Santiago de Chile

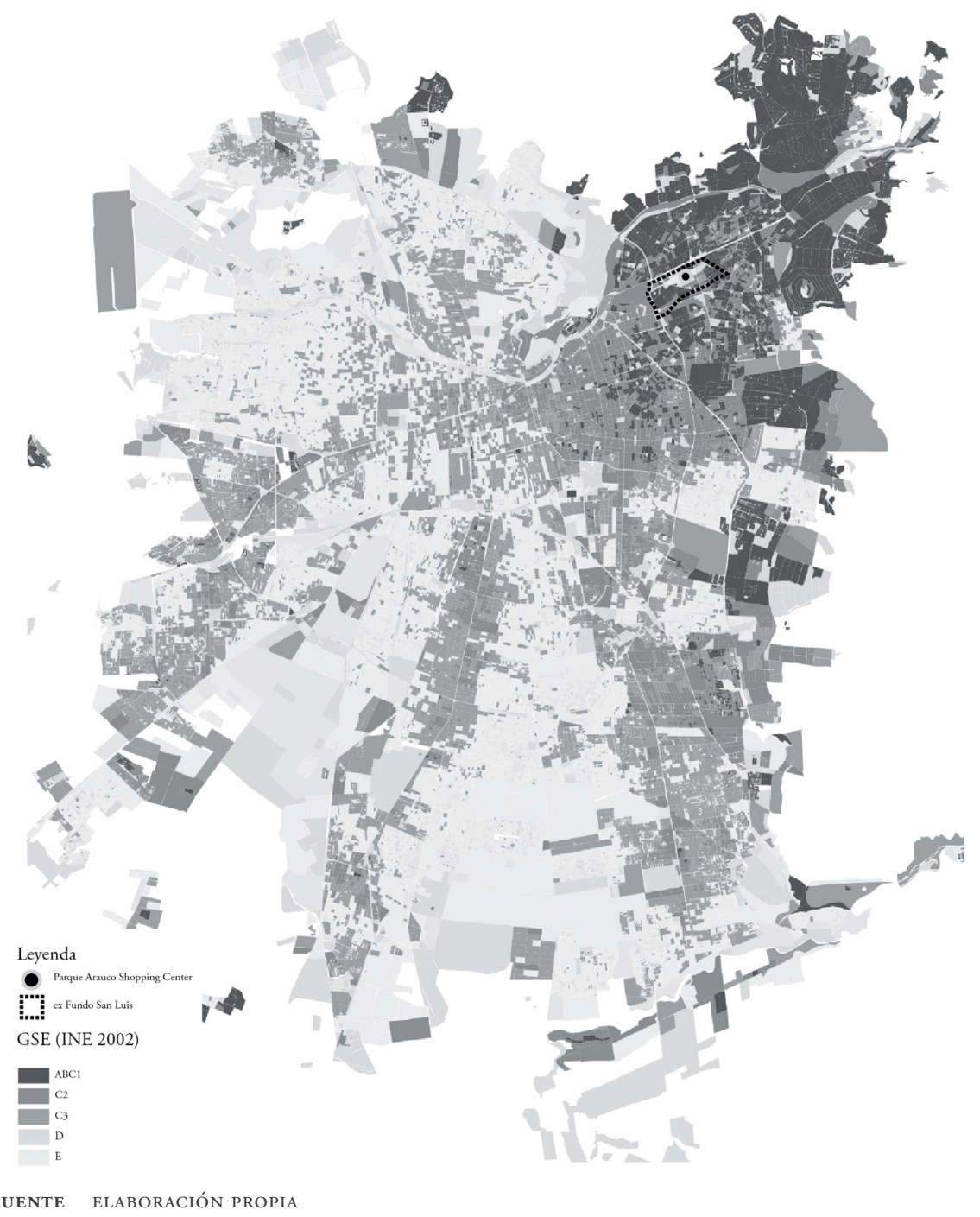

La naturalización de la infraestructura del consumo en la ciudad guarda una estrecha relación entre la arquitectura comercial importada y los discursos de modernización de la sociedad impulsados por distintos frentes. A modo de una pugna ideológica, el caso del mall en Chile muestra cómo las visiones divergentes de sociedad se fraguaron en una seguidilla de proyectos icónicos que fueron pensados para un mismo predio en el curso un siglo. La realidad edificada hoy confirma que la ciudad, como un texto, puede ser leída en sus capas de vencedores y vencidos, donde la vida cotidiana trascurre y construye nuevos imaginarios sobre los espacios urbanos (De Certeau, 1984). 


\section{El desafío de importar el mall a Latinoamérica}

Emplazado junto a avenida Kennedy (figura 1), primera autopista de la zona de alta renta, el mall Parque Arauco debuta en el Santiago de 1982 con una fórmula comercial ya conocida en otras ciudades latinoamericanas, como Caracas o Sáo Paulo: arquitectura simplificada y hermética; de líneas modernas, pero con revestimiento en materiales tradicionales; accesibilidad vehicular inmediata a las autopistas interurbanas; y lustrosas instalaciones interiores de bronce y mármol, mediadas por jardines interiores y plazoletas techadas. $\mathrm{Al}$ momento de su inauguración, sus gestores brasileńos y consultores norteamericanos proyectaban atraer a los segmentos de población de clase media y alta, que -según los estudios técnicos-iba a estar completamente motorizada una vez inaugurado el centro comercial (Nordal Associates, Inc., 1979). Los indicadores económicos auguraban un aumento explosivo en el PIB y las nuevas tendencias urbanas de finales de los setenta auguraban que la santiaguina era una población que ya se había suburbanizado lo suficiente como para ser tipificada por algunos de "semiamericanizada" (Cáceres \& Farías, 1999).

Ubicados en el cruce de grandes avenidas y troncales, tanto Parque Arauco Shopping Center como Mall Plaza Vespucio (el segundo mall chileno, 1990) convinieron en introducir un cambio radical en la tradicional tipología comercial chilena, asociada a portales, galerías y mercados. Aprovechando la nueva conectividad motorizada por sobre la accesibilidad peatonal, los primeros malls se localizaron en grandes predios que habían quedado vacíos o a medio construir en un proceso de modernización urbana iniciado en los sesenta, resultando los remanentes pericéntricos de una expansión suburbana aún no consolidada. Parecían predios suburbanos; descampados con amplias posibilidades para la expansión de los centros comerciales, pero en realidad eran pańos urbanos muy bien localizados, con accesibilidad garantizada a través de autopistas y futuras líneas ya planificadas de Metro, y con una clara vocación de densidad metropolitana que poco o nada tenía que ver con el carácter extraurbano con el que los originales shopping centers norteamericanos fueron diseñados (figura 2).

Buscando crear un modelo de negocios para Parque Arauco, los inversionistas brasileños que construyeron el Ibirapuera Shopping Mall en 1966 se aliaron con una oficina de arquitectos chilenos que ya venía experimentando formatos comerciales en torno a patios abiertos y desniveles: Bendersky y Brunetti. Analizando las prácticas culturales, haciendo alianzas políticas con el gobierno militar al mando e incluyendo a los reconocidos arquitectos locales, la compañía chileno-brasileña logró adaptar tanto morfológica como simbólicamente el "enclosed regional shopping center" "al contexto chileno.

El shopping mall fue rebautizado con un nombre que refundara su carácter foráneo y su diseño fue hibridizado con las maneras vernáculas de la arquitectura comercial peatonal. Esto no fue una novedad regional. En Venezuela, arquitectos enviados por el gobierno estadounidense fueron los encargados de construir el primer shopping mall importado. Siguiendo el modelo de retail suburbano original, 
pero adaptándolo al clima y usanza caraqueña, el primer mall de Sudamérica fue construido en el suburbio Las Mercedes en 1956. También en Argentina los arquitectos comerciales tomaron una línea similar al reciclar edificios tradicionales en el centro de Buenos Aires y convertirlos en plazas de retail. Es el caso de Abasto Shopping Center y de Galerías Pacífico, los cuales fueron construidos por arquitectos locales y consultores estadounidenses bajo un contexto político homólogo que aseguró, como en Chile, la asimilación de la sociedad de consumo bajo la tutela técnica de Estados Unidos.

\section{FIgURA 2 Parque Arauco con tienda Muricy y publicidad de Falabella en predio baldío, 1984}

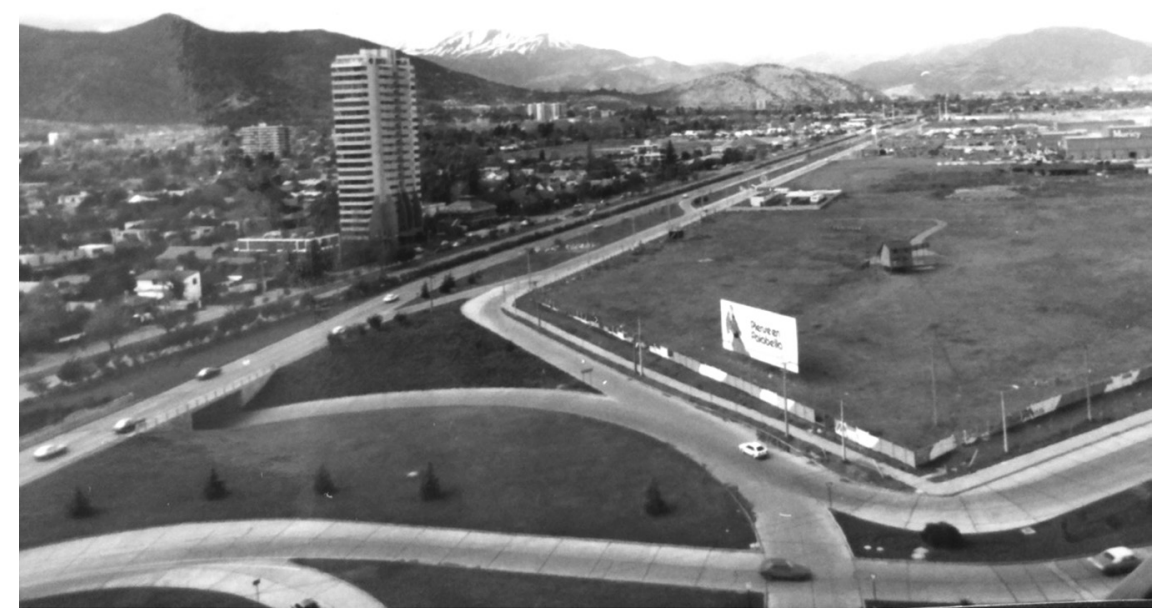

FUENTE SANTIAGOADICTO. COLABORACiÓN DE GONZALO JARA. DISPONIBLE EN HTTP://BiT.LY/2 PBLLGH

$\mathrm{Al}$ igual que en el caso chileno, los primeros malls latinos llegaron a contextos que ya tenían una larga tradición comercial. Fórmulas locales y foráneas se desarrollaron en Latinoamérica en siglos de presencia colonial primero, y en procesos de construcción republicana después. Galerías urbanas y mercados públicos, así como plazas de abastos y paseos peatonales, fueron fruto de una hibridación entre influencia extranjera y usanzas locales, con una larga historia urbana donde el intercambio siempre formó parte sustancial de la vida social urbana.

Más aun, se puede decir que en Chile el mall llegó en un contexto ya inoculado por la sociedad del consumo, y que su proclamación como un nuevo modo de vida urbana tiene dimensiones más bien ideológicas y propagandísticas. Antes que él, insinuaciones propias del consumo posmoderno -aquel que privilegia el consumo de experiencias por sobre la mera compra de productos- ya estaban presentes en los discursos publicitarios y morfológicos de los centros comerciales de los años 
cincuenta y setenta. Estas anunciaciones pueden ser advertidas ya en cuatro innovaciones previas a la llegada del mall: (i) en el modelo administrativo de los centros comerciales de mediados de siglo (que mudaron a ser espacios de manejo centralizado y no más en manos de dueños de locales (como en el caso del Centro Comercial Drugstore en Providencia, 1969); (ii) el control unitario del espacio comunitario, que buscaba gestionar los espacios comunes y atraer otros tipos de consumidores al generar atractivos alternativos a los del comercio (como fue visto en la cancha de patinaje y autocine del Centro Comercial Los Cobres de Vitacura, 1977); (iii) la incorporación de grandes tiendas departamentales como anclas para el comercio minorista (como el caso de Sears y luego Almacenes París en Plaza Lyon, 1981); (iv) o los discursos sociales con los que eran publicitados en los medios, basados en la sociabilización y reunión, eminentemente femenina y/o juvenil, que estos espacios urbanos condensaban (como el caso del Caracol Los Leones y Dos Caracoles, 1973 y 1976).

\section{Instalar el mall: discursos e imaginarios urbanos en torno al mall chileno}

La revista Ciudad y Arquitectura (CA) No18, editada por el Colegio de Arquitectos de Chile en 1977, fue dedicada a los "centros de intercambio". A finales de la década de los setenta, el entusiasmo por la apertura comercial que se prometía desde el nuevo gobierno era un discurso que llenaba de optimismo a los constructores y proyectistas. Este número evidencia el debate urbano que se desató entre los más influyentes arquitectos chilenos, y permite analizar los imaginarios que rodearon este momento en la historia de la arquitectura y planificación urbana chilenas.

El documento en cuestión se inicia con una nota editorial que proclama el escenario de la ciudad como una "verdadera fiesta del comercio [que] ha ido evolucionando junto a la especialización y diversificación de los diseños en una continuidad de escaparates y vitrinas que, de la concentración puntual [haciendo referencia al centro fundacional], pasando por el paseo longitudinal, alcanza la superficie o el volumen de los 'grandes almacenes' de hoy ('shopping o magazines')" (CA, 18, 1977, p. 4).

El editor de la revista $C A$ de la época, el arquitecto y connotado urbanista Jaime Márquez, destacaba que los nuevos complejos comerciales vendrían a suplir las nuevas demandas producidas por los cambios en la forma metropolitana. Márquez continúa: "Las diversas escalas del trasladarse en nuestra ciudad (a pie, en bus, en 'subterráneo' o en auto particular) articulan una accesibilidad que mantiene vigente diversas formas de concentrarse el intercambio de productos. (...) el comercio se separa o enlaza con otras actividades como las de esparcimiento o servicios cívicos en estos complejos elementos urbanos actuales que son los así llamados 'centros' o 'corazones de la ciudad"' ( $C A, 18,1977$, p. 4). Parafraseando el célebre título del arquitecto creador de los shopping malls en Estados Unidos, quien definiera los nuevos shopping centers como los "corazones" de una nueva planificación urbana descentralizada (Gruen, 1964; Gruen \& Smith, 1960), Márquez introduce el debate sobre el comercio urbano a través de la necesidad de contar con centros comerciales de mayor complejidad a la luz de la modernización de Santiago y sus sistemas 
transporte metropolitano. Previo a la construcción del primer shopping mall chileno, el Colegio de Arquitectos quiso orientar el debate hacia la complejidad que requería la nueva ciudad. Lejos de la mala recepción con la que hoy cuentan estos centros, los arquitectos de los setenta consideraban necesaria la transición desde el peatonal centro comercial del centro - protomalls- a nuevos complejos que respondieran a los tamańos de una metrópoli y la irrupción masiva del automóvil.

No obstante, la confusión entre las definiciones de lo que se entendía por centro comercial y por shopping center o shopping mall en los setenta en Chile descubre los imaginarios identitarios que entraron en conflicto en ese momento de gran convulsión social. Ambos conceptos no son traducciones homólogas, pues si bien shopping center es entendido en Latinoamérica como aquel edificio de origen norteamericano, climatización centralizada, mix de tiendas anclas y comercio minorista, con público marcadamente motorizado y arquitectura hermética rodeada de estacionamientos, centro comercial es una tipología de tradición latina mucho más añeja que el invento de Víctor Gruen.

Analizando la postura de otras revistas de arquitectura, ajenas al gremio institucional, resulta interesante revisar lo publicado por la revista AUCA No32, también de 1977. Adelantándose a la llegada fáctica del mall-Parque Arauco no se abriría hasta 1982, y Apumanque y Plaza Lyon, dos de los protomalls, se inauguran ambos en 1980-, el arquitecto José García Huidobro aborda su inminente llegada al contexto chileno. Para el arquitecto, el mall en otras latitudes representaba la superación de la espacialidad reducida de las grands magazins y su atomización en la ciudad (García Huidobro, 1977). Para García-Huidobro, lo que el mall ya no ofrece en materia de atractivo exterior, lo entregaría generosamente en su interior, en donde "el paso del interior hacia la calle es más fluido que en las viejas soluciones y se apoya el ascenso en rampas peatonales o escalas mecánicas. Más que una calle de locales, estos se constituyen como un lugar de encuentros, entretención y esparcimiento. Su mayor atributo es su empleo como centro comunitario y social" (García Huidobro, 1977, p. 56). Y con optimismo ante el pronto arribo del modelo a Chile, el arquitecto agrega: "el recorrido del 'mall' se convierte así en un paseo cuya reminiscencia la encontramos en los boulevares de París” (García Huidobro, 1977).

Al respecto, García-Huidobro (1977) habla de la influencia de estos nuevos centros comerciales, que "nos llegan de países desarrollados donde primero aparecen en un nivel y [luego] en varios niveles desde mediados de la década pasada (1965)" (p. 58). Es posible que los ejemplos en países vecinos ya hubiesen advertido a los arquitectos chilenos. Tanto Perú como Venezuela, Colombia, Argentina y Brasil ya contaban con sus propios malls, aunque en su mayoría localizados afuera de la ciudad (De Simone, 2017; Ward, 2005).

Sin embargo, García Huidobro habla del mall foráneo en la revista $A U C A$, pero para presentar a continuación dos proyectos de arquitectura chilena que poco o nada tenían que ver con el modelo norteamericano: Galería Drugstore (1970) y Caracol Los Leones (1973). Comparando su espacialidad interior y las actividades que allí se realizaban, el arquitecto decide ocupar el término mall, de manera inédita en las publicaciones de arquitectura y urbanismo chilenas, para referirse a una realidad eminentemente urbana, de carácter peatonal, edificios construidos en 
barrios consolidados y de diseño local (aunque uno estaba inspirado en Londres y otro en Nueva York $^{2}$ ).

García Huidobro (1977) omite cualquier tipo de crítica negativa hacia el mall. Lejos de ser una advertencia, destaca al mall por su "preocupación fundamental por los conceptos de bienestar, expresados en jardines exteriores e interiores, fuentes y espejos de agua, plazoletas, climatización total amén de satisfacer la más amplia gama de las necesidades de la población" (p. 58), cercano a la visión social que Gruen veía en el mall como unidad básica para el diseño de ciudades (Wall, 2006).

Incluso su "emplazamiento en la periferia de las ciudades" es considerado por García Huidobro (1977) un punto a su favor, pues le permite servir "tanto al centro como a las regiones adyacentes, dado que solo allí se encuentran terrenos suficientes para atender el estacionamiento masivo de vehículos, movilización característica de esas sociedades" (p. 59).

Aun cuando el shopping mall chileno existía solo en el papel, desde los años sesenta la terminología de shopping center ya estaba siendo usada para publicitar galerías comerciales, y en los setenta, caracoles comerciales. Los anglicismos usados desde el marketing urbano para promocionar los antiguos y nuevos formatos comerciales es una constatación de un cambio cultural bajo un proceso de reestructuración económica y social.

Referirse al centro comercial engloba hasta hoy las tipologías de grandes magazines, tiendas departamentales, pasajes, galerías, caracoles y portales, todas con reminiscencia europea y origen marcadamente urbano, mientras que referirse al mall es aludir directamente a lo estadounidense y su estilo masivo y suburbano. Plantear esta discusión a los pocos ańos del golpe de Estado ocurrido en Chile en 1973, y frente al modelo de política económica monetarista que trajeron luego los economistas chilenos que estudiaron con Milton Friedman en Chicago ("Chicago Boys"), implica discutir y tomar posición sobre los cambios culturales que ocurrían en el país. Más aún, esta discusión no solo enfrenta dos imaginarios sociales y posturas ideológicas en contraste, uno con ideales europeos y otro con ideales norteamericanos. El debate puso en escena a quienes acusaban de un afán refundacional al nuevo gobierno, que prometía borrar cualquier vestigio pasado y abrazar los modelos estadounidenses de economía, sociedad y vida cotidiana. A pesar de la cualidad revolucionaria que se le quiso imprimir a la llegada del mall a Chile, para muchos cronistas fue el caracol adonde se iba para "ver y ser vistos" (Providencia. 100 años de la comuna, 1997), y el mall solo cobró vida en un contexto de toque de queda y militarización del espacio público posgolpe. Los fenómenos sociales que se desarrollaron posteriormente al interior de los malls no eran novedad en el contexto santiaguino, sino que más bien se presentó al mall como una novedad propia de un nuevo modelo de sociedad.

La efervescencia del mall en Chile mucho se debe a la campaña publicitaria que lo instaló como una revolución urbana. Fue un símbolo del cambio estructural, o un estandarte de la "revolución silenciosa" que impulsaba el nuevo gobierno (Lavín, 1987).

2 Chelsea Drugstore de Londres y Museo Guggenheim de Nueva York. 
Por lo mismo, los imaginarios que rodearon al mall habrían sido construidos mediante discursos promotores, debates gremiales que desestimaron los modelos anteriores e intensas campańas publicitarias que permitieron crear una nueva idea de ciudad inaugurada con la llegada de esta tipología. En efecto, la crítica hacia el mall, iniciada más en los ochenta, tuvo un cariz dramático: no criticaba solo un edificio, o un modelo suburbano; más bien criticaba un modelo político y un marco ideológico. El mall fue visto como el blanco que permitiría desahogarse a las oposiciones al gobierno militar.

\section{“Nunca pisaré un mall": \\ lo sagrado y lo hereje en la crítica a los primeros malls}

Que las clases medias comenzaran a verse identificadas con el mall fue interpretado por los críticos de una manera dicotómica. Entre sus detractores, el consumo en el mall era consumismo exacerbado; perversión y erotización de la mercancía. El mall era la "catedral del consumo" que se apoderaba religiosamente de cualquier resquicio de toda vida pública callejera tradicional que pudiese sobrevivir (Moulian, 1999). La irrupción de Parque Arauco en los ochenta y Mall Plaza Vespucio en los noventa como espacio dominical de paseo reunió el asombro de los santiaguinos. Tal exaltación llegó incluso a declaraciones presidenciales, como la del entonces Presidente Patricio Aylwin en un noticiario televisado en 1993: "Nunca he ido ni pondré un pie en un mall', aseveró al ser preguntado si asistiría a la apertura del Alto Las Condes Shopping Center. Un año más tarde, dejaría por escrito sentir "repudio hacia ese mall que no conozco ni me interesa conocer, pues lo encuentro absolutamente desproporcionado a Chile, una ostentación al consumismo a gente que no tiene qué comer..." (Aylwin en Arroyo, 1994).

El mall como símbolo significaba un impacto moral e identitario al ascetismo aristocrático chileno, que promulgaba la reserva y solidaridad frente a la necesidad de las masas populares (Tironi, 1999).

El mall se presentaba como un espacio excepcional al que se podía ir a mirar. Es comprensible, por lo tanto, la referencia a este nuevo interior magnífico, de acceso relativamente liberado, que lo asimilaba al rol de las iglesias en la ciudad tradicional. Sus incrustaciones de bronce, relojes monumentales traídos de Holanda, pavimentos de mármol traído de Brasil, asociaron el interior del mall con aquellas atmósferas vistas antes solo en las iglesias y catedrales. Si bien las grandes tiendas de novedades chilenas de principio del siglo xx pregonaban elegancia y lujo importando, sus dimensiones jamás fueron comparables con este edificio de tres cuadras de largo que era Parque Arauco. La llegada de las tarjetas de crédito no bancario, manejadas por las nuevas tiendas departamentales, se constituyó en una promesa de ascenso social a través del consumo. Recorrer las vitrinas iluminadas, aunque no se pudiera comprar los productos importados, significó la musealización de los pasillos de Parque Arauco y sus nuevos productos importados, e impuso un nuevo régimen escópico en el contexto santiaguino: la divinización del placer de la compra ociosa, como señaló Moulian en su denominación tan elocuente. 
El primer mall chileno, que inaugurado durante la crisis económica de 1982, fue visto como espacio sagrado, un altar, una vitrina que se miraba pero no se tocaba. Más aún, un lugar al que se podía ir a conversar, pasear, jugar y no necesariamente comprar. Esto determinará la evolución de los centros comerciales en Chile. Mientras que en otras latitudes el consumo de experiencias en el shopping center se generó como una etapa posterior al consumo de productos (Crawford, 1992), en Chile la piedra angular de su éxito fue proponerse desde un inicio como un espacio limpio, seguro y confortable donde reunirse.

Inaugurado en abril de 1982, la explosión de visitas en Parque Arauco alimentó la esperanza de bonanza futura, donde el desenfreno publicitario presentaba al shopping como un proyecto cuasi civilizatorio. En efecto, para los defensores del régimen militar, la llegada de Parque Arauco fue el símbolo perfecto del triunfo del modelo neoliberal y, por lo mismo, parte integral de la articulación discursiva del libre mercado sobre la ciudad y sus habitantes, los cuales solo se podrían verse beneficiados por esta apertura (Larrain, 1996).

El gigantesco letrero que protegía las faenas de Parque Arauco meses antes de su inauguración rezaba "Aquí se construye la ciudad del futuro... hoy" (figura 3), arrogancia que fue vista por muchos como una ofensa más del nuevo modelo (Hunneus, 1979).

FIgura 3 | Cartel de las faenas de Parkennedy, 1979

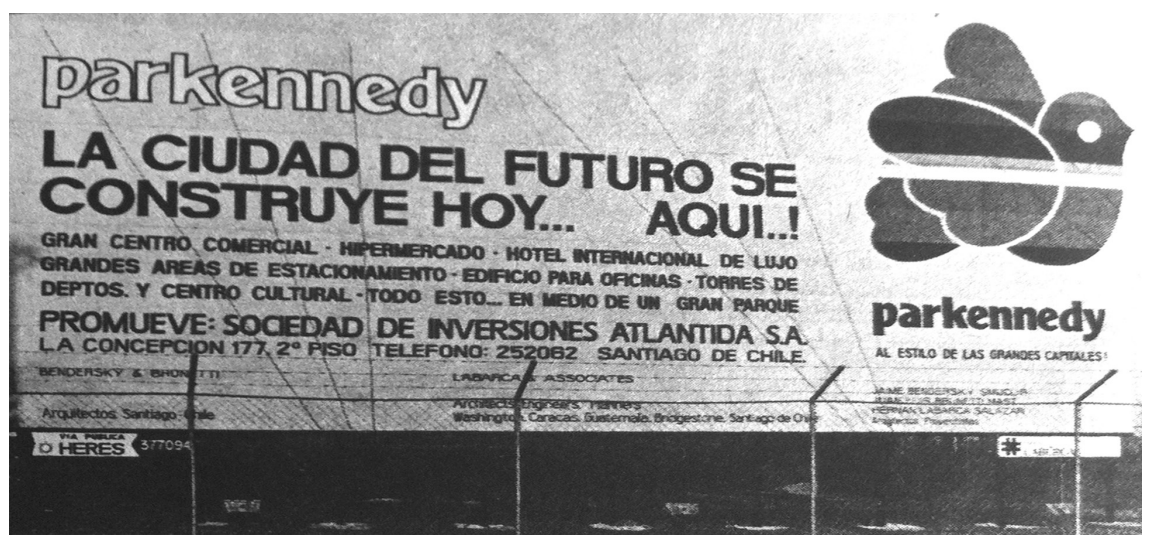

FUENTE REVISTA DEL DOMINGO, EL MERCURIO, I 979

El letrero frente a lo que sería Parque Arauco presagiaba el arribo del desarrollo urbano a través del retail. Como lo visto en otras ciudades latinoamericanas, el mall en el tercer mundo, con sus interiores inmaculados y recintos protegidos, dejaba fuera cualquier evidencia de un subdesarrollo, allí negado (Sarlo, 1998; Sato, 1981). Para muchos, en sus pasillos se creaba la atmósfera ilusoria de un simulacro urbano que tińó la experiencia urbana del latinoamericano de un halo temporal de modernidad primermundista a través del consumo. 


\section{"La ciudad del futuro se construye hoy... aquí"}

Devenido en subcentralidad de una ciudad que para la época contaba con tan solo un centro tradicional y otro lineal (eje Alameda-Providencia), Parque Arauco materializó el anhelo de convertir el cruce de las avenidas Manquehue y Kennedy en un centro alternativo al fundacional. Tanto el Plan Regulador Intercomunal de Santiago (PRIS, 1956-1960) como los proyectos de la Corporación de Mejoramiento Urbano (CORMu, 1969-1974) reconocían en esta zona, el antiguo fundo San Luis, el potencial para transformarse en un polo de densificación. Ambos proyectos pensaban para este sitio un complejo multifuncional de dimensiones antes desconocidas en la ciudad, con ideales herederos del movimiento moderno (figura 4). Fue así que, de la mano de los arquitectos Miguel Eyquem y Miguel Lawner, entre 1969 y 1973, durante el gobierno de Salvador Allende se proyectó un centro comercial y centro cívico llamado Barrio Modelo Parque San Luis, luego renombrado Comunidad San Luis (Eyquem, 1977).

En menos de diez ańos, el mismo lugar que buscaba dar cabida a un centro comercial diseñado bajo el estado del bienestar recibió el proyecto del primer shopping mall a la chilena como uno de los símbolos del crecimiento del capital privado del área más rica de la ciudad.

FIgura 4 Dibujo publicitario del proyecto de Parque Arauco

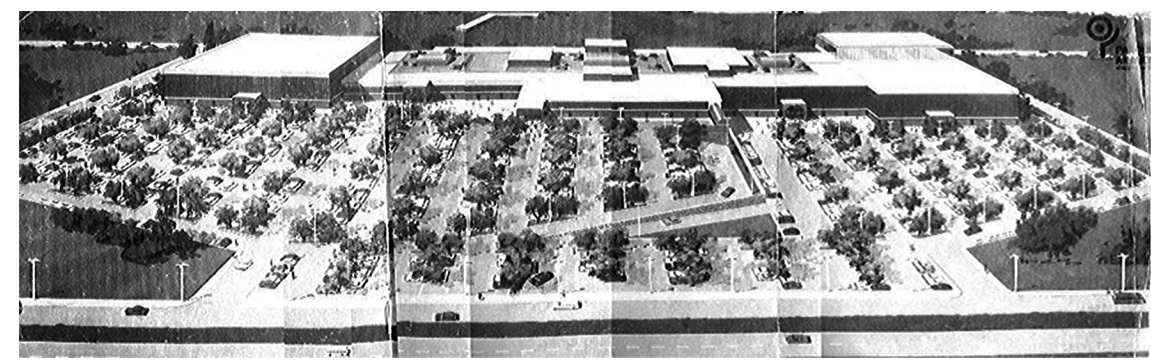

FUENTE NORDAL ASSOCIATES, INC. (I 979)

Parque Arauco Shopping Center fue presentado a la sociedad chilena como la evidencia de la modernización del Estado; de manera semejante a la manera como se presentó en países vecinos, el mall fue cubierto por la prensa como la "llegada de la Civilización”, según destaca Sato (1981) para el caso del mall venezolano.

Si en Chile hasta 1973 los grandes proyectos del Estado habían sido los depositarios de las innovaciones tecnológicas, urbanas y culturales -como, por ejemplo, el proyecto CyberSyn (Medina, 2015) o los paneles de prefabricación K.P.D. (Alonso \& Palmarola, 2015)-, el cambio radical en el sistema económico y social que generó el gobierno militar depositó las iniciativas progresistas en manos de privados, con altos ingresos económicos y contactos bancarios para la inversión estatal. De ese modo, las innovaciones urbanas de la época se vieron expresadas según los requerimientos del cliente de ese momento, y dieron fruto a tipologías de centros comerciales cada vez más experimentales, como Dos Caracoles (1973), Cobres de Vitacura (1977), 
Pueblo del Inglés (1978) y Apumanque (1980), los que constituyeron un salto en forma, formato, usabilidad y materialidad en el proyecto comercial privado.

Más que los usos comerciales y las funciones sociales, lo que trajo el shopping mall a la ciudad fue una imagen pocas veces vista antes en la capital, y que fue leída como el ícono pujante del enriquecimiento del cono alto capitalino: la gran plaza de estacionamientos. Articulada como explanada monumental, la plaza de estacionamientos llena de automóviles otorgó una visión real de lo que el "milagro económico" había traído, y que por fin empataba con las críticas que nacían contemporáneamente en otras latitudes de la región sobre la "americanización". Era aquel "centro comercial, sin ventanas ni sutilezas volumétricas, rodeado de estacionamientos" (Sato, 1981), implantado de modo suburbano al interior de una ciudad latinoamericana que, aún de manera incipiente, mostraba sus intenciones de expansión.

Aun cuando otros centros comerciales incorporaron antes un sector para estacionamientos, como Apumanque, Rampa de las Flores o el supermercado Jumbo, ninguno antes que Parque Arauco lo hizo con tanta vehemencia. La imagen publicitaria de Parque Arauco hizo uso de los estacionamientos infinitos como su mejor atractivo. "Por fin" llegaba un "verdadero" shopping center y no solo comercios vecinales que se hacían llamar como tales (figura 5).

Figura 5 | Publicidad de Parque Arauco a días de su inauguración

\section{Por fin un verdadero Shopping Center. Parque Arauco, "El primer Shopping Center de Chile."}
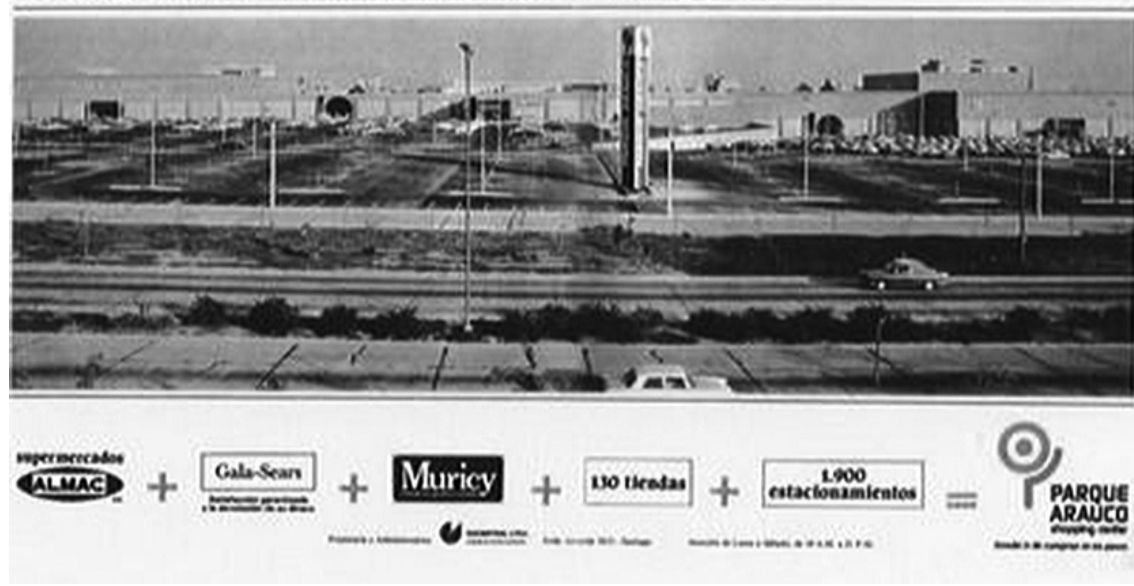

FUENTE EL MERCURIO, 24 DE MARZO DE I 982

A mediados de 1979, una consultora estadounidense contratada por los inversionistas de Parque Arauco estimaba que la renta per cápita en el área de influencia primaria del mall -es decir, aquellas zonas próximas de 5 a 7 minutos de viaje $(2,5 \mathrm{~km}$ de norte a sur en automóvil, $2 \mathrm{~km}$ dirección poniente y $5 \mathrm{~km}$ dirección oriente)- era 
un $268 \%$ mayor a la media del área metropolitana de Santiago; mientras que el área de influencia secundaria, que hacía relación a las zonas de gran comercio de la ciudad (incluye Providencia y partes del centro de Santiago), era un 407\% mayor a la media metropolitana (Nordal Associates, Inc., 1979).

No obstante, esas estimaciones no se concretaron. En 1981 estalló una crisis económica global conocida como el Efecto Tequila, que disparó la inflación y cambió por completo el escenario de apertura del mall. Más que nunca se hizo fundamental contar con el apoyo mediático y publicitario necesario para convertir a Parque Arauco en una afirmación del éxito de las políticas económicas implementadas por el gobierno militar. Y ello aun cuando esa alegoría de éxito no coincidiera con los números.

Los socios brasileños y estadounidenses se retiraron del negocio cuando estalló la crisis. Con nuevos socios chilenos (Falabella y Almacenes París), a los pocos meses de su apertura Parque Arauco buscó convertirse en un mall conectado con el Metro y el trasporte público, a fin de atraer nuevos clientes de clases medias. Los dueños del mall, ahora todos chilenos, comprendieron la necesidad de instalar un recorrido gratuito de trasporte privado que conectase la estación terminal del Metro hasta el acceso principal del centro (aprox. $4 \mathrm{~km}$ ), bus que permaneció activo hasta avanzados los noventa. Aunque proclamase una "ciudad del futuro", el primer mall de Chile tuvo que adaptarse a las necesidades de un contexto más bien peatonal, una ciudad dependiente de los buses y un barrio que siempre se pensó como subcentro metropolitano y no suburbio satélite.

\section{Un predio y cuatro imaginarios: un barrio moderno, una vivienda social, un mall suburbano y muchos rascacielos}

El fundo San Luis, lugar donde se construyó Parque Arauco, era un terreno de más de 150 hectáreas ubicado entre avenida Kennedy al norte (primera autopista de alta velocidad consolidada en Santiago) y la circunvalación avenida Américo Vespucio al poniente. El gran baldío había quedado paralizado en los ańos treinta cuando su dueña falleció y lo donó a la beneficencia, pero sus herederos objetaron el traspaso (Lawner, 1979). Siendo un vasto sitio eriazo rodeado de zonas residenciales de clase media y media alta, desde los años cincuenta el terreno había ofrecido una ubicación privilegiada para cualquier proyecto urbano de gran envergadura, aunque en esos momentos albergara algunas tomas y campamentos.

Durante el gobierno de Eduardo Frei Montalva (1964-1970) las tierras del fundo San Luis fueron expropiadas. La CORMu pensó para el lugar un proyecto revolucionario. Se trataba de una intervención urbana sin precedentes a nivel nacional, una verdadera "ciudad dentro de la ciudad", planificada y ejecutada por el Estado (Pulgar \& Bianchini, 2008, p. 32). Fue así que se ideó uno de los más grandes proyectos urbanísticos de la época, el Barrio Modelo Parque San Luis (figura 6). En él se proponía ubicar a familias de ingresos medios y medios-altos, un total de 70.000 habitantes y 150 hectáreas de intervención. A través de 61 torres de 17 a 20 pisos, y 40 edificaciones de 4 a 5 pisos, el arquitecto Miguel Eyquem se proponía realizar un conjunto urbano de gran escala, que incluyera en su interior un centro 
cívico y la nueva sede consistorial de la Municipalidad de Las Condes. El edificio, pensado como una sede cultural y comercial, pretendía tener dimensiones muy similares a lo que luego sería el pabellón del primer mall que se construiría en el mismo lugar. El centro reuniría espacios de entretención, servicios y comercio, y tendría que incorporar tecnologías que permitieran una gran flexibilidad. Se llamó a un concurso público para su diseńo, de carácter revolucionario: no existían precedentes de tamaña infraestructura cultural para un subcentro en Chile.

Al centro del proyecto urbanístico se proyectó un gran parque lineal (Parque Araucano). En su interior se propuso un amplio sector destinado a instalaciones deportivas, concedido para su desarrollo a la Corporación de Fútbol de la Universidad de Chile (Corfuch). El club deportivo se comprometió a desarrollar un estadio de fútbol para 15.000 personas y una piscina olímpica, aceptando servir gratuitamente de infraestructura deportiva a las familias que vivirían en el sector.

\section{FIgURA 6 | Planta general de urbanismo conjunto habitacional Parque San Luis}

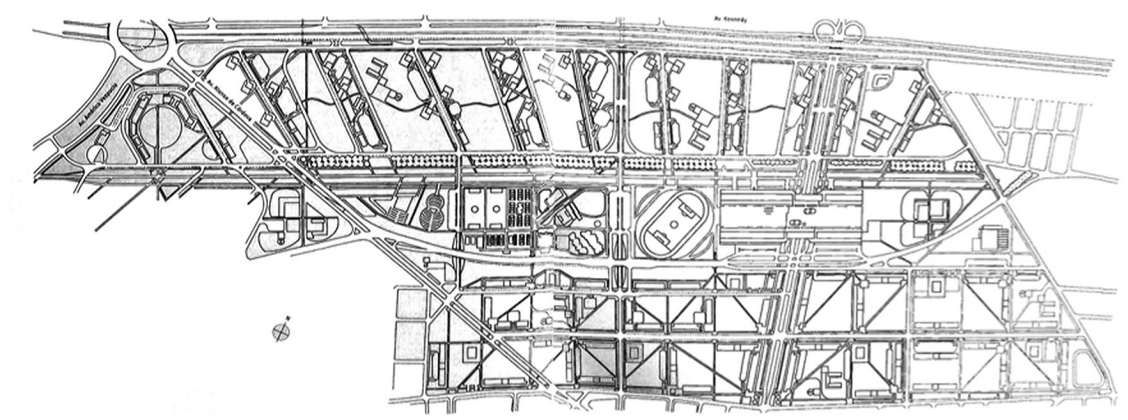

FUENTE EYQUEM (I998)

Sin embargo, cuando en 1970 fue elegido Salvador Allende a la presidencia de la república, la política urbana se delineó radicalmente hacia la integración social, por lo que un "un proyecto de tal naturaleza resultaba antagónico con el programa del gobierno de Allende" (Lawner, 1979, p. 2). En 1971, la cormu, con nuevo director ejecutivo, Miguel Lawner, replanteó el proyecto del fundo San Luis. Los destinatarios ya no serían de clase media y media-alta, sino que se daría vivienda a los pobladores de campamentos de la misma zona de Las Condes. Lawner y su equipo vieron el megaproyecto urbano de San Luis como una oportunidad para abastecer a la capital de vivienda social.

El proyecto pasó a llamarse Villa Compañero Ministro Carlos Cortés, en nombre del fallecido ministro de Vivienda de la Unión Popular, quien había encabezado las gestiones para asignar el proyecto original a los "sin casa" de Las Condes. Dada la premura de las exigencias habitacionales, la CORMu convocó a los talleres de las Escuelas de Arquitectura de la Pontificia Universidad Católica y de la Universidad de Chile, con el motivo ańadido de fortalecer las relaciones con las universidades. Entre 1971 y 1972 se construyeron en la zona sur bloques de cuatro pisos que albergaron a más de 1.038 familias. La Villa ofrecía viviendas de dos y tres dormitorios de entre 
60 y 80 metros cuadrados. Algunos bloques habitacionales fueron diseñados por arquitectos de renombre y sus estudiantes: Sergio Larraín García-Moreno, Ignacio Covarrubias, Jorge Swinburn, Alberto Collados, Nicolás Freund, Gonzalo Leiva e Isidoro Loi, todos con una fuerte influencia del movimiento moderno (Lawner, 1979, p. 9).

Los pobladores, además de cumplir con los pagos que la CORMU exigía para otorgar la propiedad de los inmuebles, participaron extensivamente del proceso de diseño y construcción, marcando un precedente en los proyectos urbanísticos en Chile.

En 1972, la zona norte del proyecto, que iba a ser urbanizada por 61 torres, fue suspendida del proyecto de la nueva CORMu. Al quedarse sin edificaciones hacia septiembre del 1973, la zona quedó baldía y, paradójicamente, pudo ser ocupada seis años más tarde por el mall Parque Arauco (salvo tres edificios que alcanzaron a ser construidos; figura 7).

FIGURA 7 Infografía antes y después, ex fundo San Luis

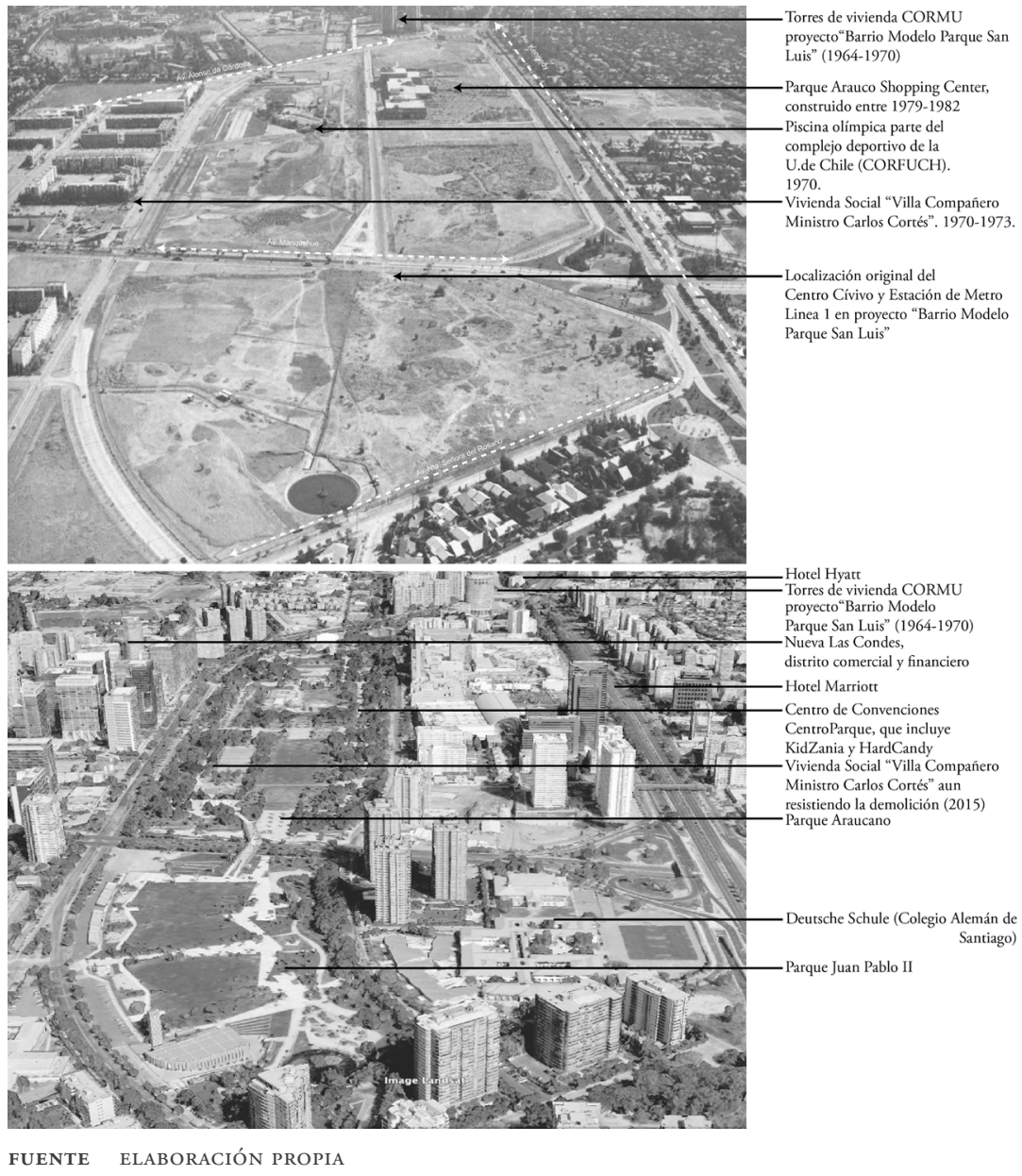


El sector fue renombrado Villa San Luis. Se dispuso control militar en la zona y los departamentos fueron reasignados a suboficiales del Ejército. A las familias desalojadas les fueron desconocidos sus pagos y derechos por sobre la vivienda que habían adquirido legalmente. Como relata Lawner (2008), fueron desalojados de noche y llevados en camiones a predios del borde de la ciudad. En 1979, la sociedad conformada por Thomas Fürst, la familia Said, los arquitectos Benderky y Brunnetti, y una firma de inversionistas brasileños (la multinacional paulista СврI Internacional), compraron el tercio norte del ex fundo San Luis que iba desde avenida Américo Vespucio a avenida Manquehue. Los integrantes del grupo conocían el proyecto de la CORMu de Eyquem y concordaban en que este debía ser un polo de desarrollo urbano ajustado a las premisas de la planificación metropolitana previa. Tenían un gran proyecto inmobiliario en mente, con 19 edificios de departamentos, además del centro comercial con dos tiendas ancla e interior climatizado, idea bastante similar al centro cívico y Comercial de la cormu de los años setenta. En la más grande inversión internacional hecha en Chile hasta ese entonces, los fondos prestados por el Banco de Brasil (27 millones de dólares) financiaron la "más grande operación inmobiliaria de los últimos tiempos” (Cummings, 1982, p. 5). A cambio, los materiales de construcción tenían que ser traídos desde Brasil y vendidos por empresas brasileñas.

Sin embargo, con la crisis económica de los años 1981-1982 se debió entregar una sección del predio en parte de pago a los bancos (Said, 2004). Solo se pudo construir el pabellón inicial, con estética suburbana de caja cerrada, accesos exclusivamente hacia el norte (desconociéndose el parque y la villa) y una amplia plaza de estacionamientos que presagiaba el nuevo paisaje que se instalaría en la zona, muy lejano al boyante subcentro que una década antes Eyquem había pensado.

En cuanto al resto del ex fundo San Luis, la historia es menos conocida, pero más evidente. En 1996, el Ejército de Chile vendió los terrenos de la Villa San Luis, a través de una licitación privada, a la Sociedad Inmobiliaria Parque San Luis, en una suma que ascendió a los ochenta millones de dólares. Se procedió a derribar los edificios. El alcalde de Las Condes de la época, Joaquín Lavín, montado sobre una retroexcavadora, abrió literalmente el paso a lo que se veía como una nueva etapa en el futuro de la comuna, allí donde nuevamente se construiría "la ciudad del futuro". La inmobiliaria ya se publicitaba en 1997 como la responsable de uno de los proyectos inmobiliarios más grandes del país, Nueva Las Condes, lugar hoy de las oficinas más caras y modernas de Chile.

Con respecto al estadio de la Universidad de Chile, lo único que se alcanzó a construir fue la piscina. Las faenas de excavación del estadio de fútbol, cuyo diseño sería semienterrado y en forma de talud verde, iban a iniciarse en septiembre de 1973. Hoy, el sector central sigue siendo un parque, pero alberga uno de los centros de convenciones subterráneos más grandes de la capital (CentroParque), junto con un centro recreativo-comercial para nińos (KidZania) y un gimnasio de cadena global (HardCandy, de propiedad de la cantante Madonna).

El PRIs de 1960 había reconocido la zona como el futuro subcentro oriente de la capital, siguiendo criterios de descentralización que buscaban modernizar Santiago. Junto con la zona del actual mall Plaza Vespucio en la comuna de La Florida, el 
subcentro del Parque Araucano es uno de los pocos que se logró consolidar. Tras la desintegración del interés del Estado por impulsar este tipo de proyectos, solo las inversiones privadas pudieron llevarlos a cabo. Los centros de servicios e intercambio, materializados en centros comerciales tipo mall (como Parque Arauco y Plaza Vespucio), evidencian la interpretación que hizo el mercado de las subcentralidades que la planificación urbana estatal había ideado. El proyecto de la CORMU iba a ser la materialización estatal de un subcentro que solo fue desarrollado veinte años después por privados, el retail y la especulación inmobiliaria.

\section{Discurso y ciudad: el simbolismo del mall “a la chilena”}

Pese al contexto económico recesivo inicial, el mall Parque Arauco simbolizó la confirmación de un proyecto nacional en vías de éxito. Si bien su plan inicial (19781979) desbordaba de entusiasmo inmobiliario, promovido por la liberalización del mercado de suelo, tal impulso se vio truncado por la crisis. El diseńo original, con sus torres de vivienda, centros culturales, hoteles y parques en el pańo superior del ex fundo San Luis, se vio reducido a un pabellón comercial lineal. De 8 metros de altura y 300 de largo, el mall quedó sumido en un mar de 2.000 estacionamientos asfaltados y otros tantos metros cuadrados de terrenos baldíos, que de algún modo fungían como la promesa futura de la realización del proyecto original.

Sin embargo, Parque Arauco Shopping Center no solo vio mermado su proyecto. La polémica por su arribo provocó también que tuviese que desistir de otras cosas, como su nombre original: Parkennedy.

Con el imponente letrero que proclamaba "la ciudad del futuro", Parkennedy, se publicitaba la creación de un "gran centro comercial. Hipermercado. Hotel internacional de lujo. Grandes áreas de estacionamiento. Edificios para oficinas y centro cultural. Todo esto en medio de un gran parque" (Hunneus, 1979). Los planos contemplaban 19 edificios rectangulares alineados en donde se proyectaban más de 2.000 departamentos y 2.600 automóviles, en total 70.000 metros cuadrados y 400 millones de dólares (Hunneus, 1979).

Desde la prensa nació la polémica por el nombre anglosajón del proyecto. La Revista del Domingo (El Mercurio), dirigida por el periodista Luis Alberto Ganderats, recalcó la atopia del nombre importado, el que no consideraba las múltiples significaciones que había tenido ese terreno.

El alcalde de la Municipalidad de Las Condes en ese momento, Alberto Labbé, propuso rebautizarlo como Parque Lautaro, en homenaje al gran estratega militar indígena. ${ }^{3}$ El equipo de periodistas sugirió Parque Arauco, recogiendo el nombre literario universal del pueblo mapuche. ${ }^{4}$ Finalmente, en agosto de 1979, la Revista del Domingo da cuenta del cambio del nombre Parkennedy a Parque Arauco como una gran batalla ganada, y publica un texto de su director en que se deja constancia de las motivaciones de esta campaña contra el anglicismo (Ganderats, 1979).

3 Nombres mapuche fueron empleados por miembros gobierno militar para renombrar calles y avenidas.

4 La Araucana (1569-1589) es un poema épico del español Alonso de Ercilla que relata la primera fase de la Guerra de Arauco entre espańoles y mapuches o araucanos. 
El discurso del primer mall en Chile propugnó un proyecto que incluía a todos los miembros de la sociedad, fuesen estos consumidores o no. Su novedad radicó en su planteamiento como un proyecto de renovación social, pues prometía la creación de una nueva manera de habitar la ciudad, que convocaba a todos los miembros de la sociedad a participar de su debate. En este proyectar el futuro de la vida en la ciudad, el automóvil, el consumo y el imaginario vencedor (Arauco y La Araucana) fueron temas que la apertura del primer mall chileno puso en debate. Tanto oponerse a su simbolismo como celebrar su triunfo en la ciudad era expresar una postura ideológica sobre la realidad pasada y futura del país.

Es probable que haya sido esta condición atemporal del discurso en torno al mall Parque Arauco la que influyó en las primeras valoraciones arquitectónicas -y ya no semánticas- de su edificio. La historia del predio y su significado en la lucha histórica de los distintos flancos políticos y sociales permeó la valoración que se hizo del proyecto comercial en sí mismo. Los deudos del proyecto social de la Unidad Popular se vieron traicionados en sus valores, mientras que quienes añoraban la etapa previa, aquella de la CORMu y su poder modernizador, se sintieron rechazados frente a un proyecto importado y ajeno a la realidad local.

Más aún, los discursos publicitarios que pretendieron presentar el mall como una victoria que afianzaba un futuro de progreso y bonanza a través de un espacio de consumo motivaron a las voces que se opusieron tanto a su simbolismo como a su arquitectura comercial. A pesar de que la obra de Bendersky y Brunnetti era aclamada antes de esta construcción, después del mall recibió numerosas críticas. Al respecto, en 1977 el arquitecto Eduardo San Martín declaró sobre el Parque Arauco, aun sin construir, que "lo único que permanece es el cambio [y] en ese sentido el edificio [Parque Arauco] trasciende el tiempo (...). Puede ser que el 'shopping' de Bendersky en diez ańos más no sea eso. Puede que esa misma estructura albergue una actividad absolutamente distinta, cultural o recreativa" (CA, 18, 1977, p. 34).

El "shopping de Bendersky", rodeado de baldíos, prometía la latencia de un desarrollo futuro, uno que incluso pudiese devolver la vocación social al predio San Luis.

Los arquitectos Bendersky y Brunetti buscaron imprimir en este pabellón multiprogramático los ideales del regionalismo modernista. Si bien el partido general del mall alude al más puro funcionalismo - la función interior determina la forma pura del volumen exterior-, los arquitectos buscaron imprimir una "escala humana" en donde pudieron, o allí los dejaron. El trabajo de fachadas, con una modulación que hace resonancia con ritmos neoclásicos, y el enchape en ladrillo, buscaron afinar la gran escala desproporcionada con una medida humana.

Diez años después de la apertura del mall, cuando este ya había sido adulado y vilipendiado por la sociedad chilena, Bendersky publicó en la revista del Colegio de Arquitectos (CA) una reseńa de este "logro frente a la adversidad del encargo" (Bendersky, 1993) . Para los arquitectos, señalaba, "entre las recomendaciones (casi reglamentarias), el shopping center no debía ser atractivo por fuera, produciéndose al interior el mayor despliegue de incentivos visuales. Además, los espacios comunes no debían superar el 30\% de la superficie construida, por cuanto el shopping center es una inversión de arrendamiento” (p. 40). Es evidente la desazón de Bendersky frente a esta anulación de la obra en pos del negocio. La fachada del mall, casi como 
un lienzo, ${ }^{5}$ será el punto más expresivo de la propuesta. A través de las incisiones en la cornisa superior, el mall propuso una obra monumental, pero con posibilidad de ser medida y contrapuesta a la cordillera a modo de farellón, como describe Bendersky (p. 41).

Las referencias a los arcos en los zaguanes aluden a una condición tradicionalista, al igual que la elección del ladrillo, elegido por su "envejecimiento con nobleza". La arquitectura de "la ciudad futuro" podría ser indiferente a su contexto y la carga histórica de su emplazamiento, pero a vista de sus arquitectos, el ladrillo mantendría una nobleza histórica frente a un paisaje dramático, tanto de la Villa San Luis como de la cordillera de Los Andes. "Este tratamiento consigue un objetivo importante: cual es el de quitarle brutalismo a esta masa volumétrica tan importante" (Bendersky, 1993, p. 41).

Aun hoy, es ineludible la importancia del predio donde se emplazó el mall. Como recalcaba Santiago Cummings, director de Cocentral (Compañía de Centros Comerciales S.A.), la inmobiliaria responsable de construir Parque Arauco, hubo "cuatro" condiciones para poner un proyecto como este en ese lugar: "primero ubicación, segundo ubicación, tercero ubicación y cuarto ubicación” (Cummings, 1982, p. 5). Reflexionando una década después, Bendersky (1993) reconoce que el fundo San Luis, sumido como una "isla en la ciudad", hacía evidente pronosticar que el mall sería un éxito y que el proyecto se ampliaría hasta convertirse en un polo de desarrollo fundamental para la configuración de la ciudad (p. 40).

Frente a tanto entusiasmo, la prensa se preguntaba, antes de la inauguración, si todo este tamańo no sería desmedido. En una entrevista al ingeniero brasileño August Berger, jefe de obra de la construcción del mall, al ser cuestionado por "la chilenidad" del proyecto importado, él respondía que era justamente en la materialidad de ladrillo y hormigón, y en los arcos de medio punto, de inspiración española según el entrevistado, que se reunía el carácter criollo del mall (Berger, 1981). Criollo, aun cuando el mármol en sus pisos, el granito rojo de los pilares, fueron todos traídos de Brasil, y el reloj de campanas de la plazoleta principal, de Holanda. Incluso, en un desaire a la participación de los arquitectos nacionales, el ingeniero declaraba que "el concepto arquitectónico fue forjado en Brasil, por lo que tiene una componente latina”. 6

¿Qué hay de chileno en el primer mall en Chile? Un diseño de ladrillo en la fachada y la adecuación de un pabellón prediseńado a un terreno particular y simbólico de la historia reciente chilena.

Desde el visionario PRIS de 1960 hasta el actual proyecto comercial en altura Nueva Las Condes pasaron cuarenta años de políticas urbanas motivadas por múltiples ideologías, la mayoría de las veces contradictorias. Sin embargo, el subcentro planteado se concretó desde la instalación del mall (Poduje, 2006) y paradójicamente sigue en desarrollo con la incorporación de rascacielos, torres de oficinas y vivienda en altura.

5 Jaime Bendersky es también reconocido por su obra pictórica.

6 Y este hecho queda comprobado al revisar los actuales planos de recepción de obra presentes en la Municipalidad de Las Condes, los cuales están detallados en portugués, con traducciones al español hechas por Bendersky y Brunnetti. 
En sus más treinta años, Parque Arauco Shopping Mall ha ejecutado múltiples modificaciones a su proyecto original, abriendo sus bordes, haciéndolo más permeable e incluso integrando el Parque Araucano. Además, desde los noventa ha ejecutado ampliaciones hasta llegar a cuadruplicar su superficie inicial y ha sumado una torre clínica, dos megatorres de oficinas y en 2019 inaugurará un rascacielos con hotel, residencias y oficinas de lujo.

Paradójicamente se estaría rescatando el ideal de la CORMU, que proponía torres de vivienda en densidad. Capa tras capa, el fundo San Luis es el recipiente de ejercicios que proponen visiones divergentes de futuro.

En la historia del fundo San Luis es posible reconocer lo que Sabatini y Arenas (2000) postulan con respecto a las "resonancias geográficas", concepto por el cual entienden el efecto de perpetuación en el tiempo de un cierto orden o forma espacial, más allá de la vigencia del sistema de relaciones urbanas que le dio origen, sea este económico, cultural o de gestión pública. La herencia del Estado intervencionista -Frei Montalva y Allende- y su capacidad de manejo del futuro de un predio de tal magnitud produjo en el fundo San Luis un espacio de borde entre diversas ideologías políticas (Sabatini \& Arenas, 2000) y entre diversas identidades de lo chileno. Es esto lo que hizo tan simbólica la inauguración del mall, pues fue el ícono de la instalación del mercado neoliberal en Chile y de la apertura nacional hacia referentes no locales. El mall en San Luis evidenció la batalla no solo por un terreno ganado, sino por una memoria conquistada y luego arrasada: la de Allende, por un lado, pero también la del Estado como constructor de ciudad, en un plano más general.

En los proyectos que se han fraguado y disuelto en San Luis se leen los diversos discursos que han marcado el desarrollo urbano de Santiago. Las políticas de modernización centralizada por parte de los gobiernos de 1950-1973, que bajo su mando remodelaron la imagen de la ciudad, dejaron huellas difíciles de demoler, ${ }^{7}$ y que aún hoy interpelan a un presente urbano que carece de idealismo visionario en sus prácticas. Por su parte, la política de liberalización de los mercados de suelo urbano y la política de vivienda social del período libremercadista en 1975-1990 revoluciona -no tan silenciosamente- el orden espacial establecido por el período anterior. De ese modo, la resonancia geográfica proveniente del intervencionismo es perpetuada por las políticas neoliberales en la misma línea, pero se traslada desde la búsqueda por inclusión vía residencia social hacia la inclusión vía acceso al consumo de bienes. Ejemplo de esto sería el planteamiento del subcentro cívico del PRIS con el posterior desarrollo de un megamall que sigue expandiéndose y diversificando su oferta.

El fundo San Luis, paradigmático trozo de la ciudad, moldeado por tantos ideales contradictorios de sociedad, es el protagonista de un período que estremece por su radicalidad ideológica. Palimpsesto de discursos edificados, es la evidencia material de la transformación de una sociedad y de la gravitación del consumo como fuerza productora de lo urbano.

7 Posterior a su aceptación (abril, 2017), a la fecha de la corrección de este artículo (julio, 2017) el Consejo de Monumentos Nacionales declaró los edificios de vivienda social remanentes en el fundo San Luis como Monumento Histórico y dictó la conservación de los edificios como ícono de la inclusión social (28 de junio de 2017), deteniendo su demolición y congelando los permisos de edificación que habían sido aprobados. Más información en http://www.latercera.com/noticia/ villa-san-luis-declarada-monumento-historico-nacional/. 


\section{Referencias bibliográficas}

Alonso, P. \& Palmarola, H. (2015). Trayectorias de un panel. ARQ, (90), 34-41. https://doi. org/http://dx.doi.org/10.4067/S0717-69962015000200005

Arroyo, G. (1994). Patricio Aylwin se confiesa con revista Mensaje. Mensaje, 426, 27-35.

A.T. Kearney. (2016). The 2016 Global Retail Development Index: Global Retail Expansion at a Crossroad). http://bit.ly/1TXsRhQ

Bendersky, J. (1993). Parque Arauco Shopping Center. Revista CA, 72, 40-45.

Berger, A. (1981, noviembre 11). Entrevista a August Berger. El Mercurio.

Cáceres, G. \& Farías, L. (1999). El espacio urbano: efectos de las grandes superficies comerciales en el Santiago de la modernización ininterrumpida, 1982-1999. Ambiente y Desarrollo, 15(4), 36-41. https://doi.org/10.3900/fpj.7.6.380.s

Ciudad y Arquitectura (CA). Revista oficial del Colegio de Arquitectos de Chile, Nº18 (1977). "Diálogo: tópico 10, primera parte: centros de intercambio", pp. 30-36. http:// revistaca.cl/revistas/018.pdf

Crawford, M. (1992). The world in a shopping mall. En M. Miles (ed.), The city cultures Reader (pp. 125-140). Londres: Routledge.

Cummings, S. (1982, abril 2). Entrevista a Santiago Cummings. El Mercurio.

De Certeau, M. (1984). The practice of everyday life. Berkeley, CA: University of California Press.

De Simone, L. (2015). Metamall: espacio urbano y consumo en la ciudad neoliberal. Santiago de Chile: Colección Estudios Urbanos y Ril editores.

Eyquem, M. (1977). Centro comercial, centro cívico. CA, 18, 20-25. http://revistaca.cl/ revistas/018.pdf

Eyquem, M. (1998). El Centro Oriente según la CORmu: 1970-1974. ARQ, 40, 42-43. http:// www.edicionesarq.cl/1998/arq-40-grandes-urbanizaciones/

Ganderats, L. A. (1979, agosto 12). Editorial. Revista del Domingo. El Mercurio.

García Huidobro, J. (1977). Desarrollo del comercio. AUCA, 32, 55-59.

Gruen, V. (1964). The heart of our cities: The urban crisis, Diagnosis and cure. Nueva York: Simon and Schuster.

Gruen, V. \& Smith, L. (1960). Shopping town USA: The planning of shopping centers. Nueva York: Van Nostrand Reinhold.

Hunneus, P. (1979). Lo comido y lo bailado... Ensayos sobre la vida misma. Santiago de Chile: Editorial Renacimiento.

Iribarne, P. \& Friedmann, S. (1984). Ayer caracoles, hoy gasolineras. AUCA, 48, 21-23.

Larraín, C. (1996, septiembre 4). El "mall” y el mal. El Mercurio, p. 2.

Lavín, J. (1987). Chile, revolución silenciosa. Santiago de Chile: Zig-Zag.

Lawner, M. (1979). Demolición de la Villa San Luis en Las Condes, historia de dos despojos. Copenhague: Centro de Estudios Nacionales de Desarrollo Alternativo (CENDA).

Lawner, M. (2008, diciembre 15). Cartas al director: La triste historia de la Villa San Luis. El Mercurio.

Medina, E. (2015). From cybernetic revolutionaries: Technology and politics in Allende's Chile. Review: Literature and Arts of the Americas, 48(1), 109-117. https://doi.org/10. 1080/08905762.2015.1021134

Moulian, T. (1999). El consumo me consume. Santiago de Chile: Libros del Ciudadano. 
Nordal Associates, Inc. (1979). Market demand analysis. Parque Arauco Shopping Center: el primer shopping center de Chile. Nueva York.

Poduje, I. (2006). El globo y el acordeón: planificación urbana en Santiago: 1960-2004. En A. Galetovic (ed.), Santiago: ¿dónde estamos?, ¿hacia dónde vamos? (pp. 231-276). Santiago de Chile: Centro de Estudios Públicos.

Providencia. 100 años de la comuna (1997). Santiago de Chile: Ediciones de la Esquina. http:// www.memoriachilena.cl/602/w3-article-62134.html

Pulgar, C. \& Bianchini, M. (2008). Villa San Luis de Las Condes: lugar de memoria y olvido. Repositorio Académico - Universidad de Chile, 29-40. https://dx.doi.org/10.5354/07195427.2008.28163

Sabatini, F. \& Arenas, F. (2000). Entre el Estado y el mercado: resonancias geográficas y sustentabilidad social en Santiago de Chil. EURE, 26(79), 95-113. https://dx.doi. org/10.4067/S0250-71612000007900006

Said, J. (2004, enero 17). José, el grande. El empresario revisa su historia y la del país. Revista El Sábado. El Mercurio. http://diario.elmercurio.com/detalle/index.asp?id=\{3c6f5382b22f-4668-b6f4-6b6b3bf66d6f \}

Salcedo, R. \& De Simone, L. (2013). Una crítica estática para un espacio en constante renovación: el caso del mall en Chile. Atenea [Concepción], (507), 117-132. https:// doi.org/10.4067/S0718-04622013000100008

Sarlo, B. (1998, marzo 22). El centro comercial. Diario La Jornada Semanal. http://www. literatura.org/Sarlo/bscentro.html

Sato, A. (1981). Un simulacro urbano. Revista Punto [Facultad de Arquitectura y Urbanismo, Universidad Central de Venezuela], 19(63), 25-35.

Tironi, E. (1999). La irrupción de las masas y el malestar de las elites: Chile en el cambio de siglo. Santiago de Chile: Grijalbo-Mondadori.

Wall, A. (2006). Victor Gruen: From urban shop to new city. Barcelona: Actar.

Ward, E. R. (2005). El diseño de centros comerciales en América. RA. Revista de Arquitectura [Universidad de Navarra], 07, 71-82. http://dadun.unav.edu/handle/10171/18030 